\title{
Collective atomic recoil lasing including friction and diffusion effects
}

\author{
G. R. M. Robb, ${ }^{1}$ N. Piovella, ${ }^{2}$ A. Ferraro, ${ }^{2}$ R. Bonifacio, ${ }^{2}$ Ph. W. Courteille, ${ }^{3}$ and C. Zimmermann ${ }^{3}$ \\ ${ }^{1}$ Department of Physics, University of Strathclyde, Glasgow, G4 ONG, Scotland \\ ${ }^{2}$ Dipartimento di Fisica, Università Degli Studi di Milano and INFM, Via Celoria 16, I-20133 Milano, Italy \\ ${ }^{3}$ Physikalisches Institut, Eberhard-Karls-Universität Tübingen, Auf der Morgenstelle 14, D-72076 Tübingen, Germany
}

(Received 3 November 2003; published 27 April 2004)

\begin{abstract}
We extend the collective atomic recoil lasing (CARL) model including the effects of friction and diffusion forces acting on the atoms due to the presence of optical molasses fields. The results from this model are consistent with those from a recent experiment by Kruse et al. [Phys. Rev. Lett. 91, 183601 (2003)]. In particular, we obtain a threshold condition above which collective backscattering occurs. Using a nonlinear analysis we show that the backscattered field and the bunching evolve to a steady state, in contrast to the nonstationary behavior of the standard CARL model. For a proper choice of the parameters, this steady state can be superfluorescent.
\end{abstract}

DOI: 10.1103/PhysRevA.69.041403

PACS number(s): 42.50.Vk, 34.50.-s, 42.55.-f, 42.60.Lh

The mechanical effect of light on atoms has now been the subject of intense theoretical and experimental research efforts for several decades. However, the fact that the collective atomic center-of-mass motion of atoms can strongly influence the evolution of optical fields has only received attention relatively recently [1-4]. Recent experimental studies involving large numbers of cold atoms in high quality cavities [5-7] represent an important advance in this field, allowing detailed experimental studies of collective atomlight interaction dynamics. During these interactions both the mechanical effect of the cavity modes on the atomic motion and the driving of the cavity modes by the dynamic spatial distribution of atoms in the cavity must be described selfconsistently and cannot be considered independently.

A recent experiment by Kruse et al. [5] represents the unambiguous realization of the collective atomic recoil lasing (CARL) model originally proposed by Bonifacio and coworkers [1], which describes collective backscattering of an optical pump field by a sample of cold atoms. Previous experiments on CARL have been performed in hot atomic vapors $[8,9]$ where, however, the gain of the backward field cannot be unambiguously attributed to atomic recoil.

Here we extend the previous theoretical work on CARL, including the effects of friction and diffusion forces acting on the atoms due to the presence of optical molasses fields. We describe the system using a set of coupled Maxwell-FokkerPlanck equations. A linear stability analysis reveals that there is a threshold condition for the pump power above which collective backscattering occurs. Preliminary experimental results confirm this prediction [10]. We further show that the backscattered field and atomic density modulation amplitude or "bunching" evolve to a steady state, in contrast to the nonstationary behavior predicted by the standard CARL model. Our model describes the main features of the experimental results of Ref. [5].

In addition to the so-called "good-cavity" regime in which the experiment of Ref. [5] operates, we also examine the behavior of the system in the "bad-cavity" regime. We show that in this regime the atoms emit in a superfluorescent way [11], with scattered intensity $\propto N^{2}$, where $N$ is the number of atoms. This is a unique example of a steady-state superfluo- rescence, i.e., superradiance from an incoherently prepared atomic system.

Our system consists of an ensemble of $N$ atoms backscattering a far-detuned pump field into a counterpropagating mode of a ring cavity, described by a set of equations derived by Bonifacio and co-workers [1-3]. The equations are here generalized to include a friction force $-\bar{\gamma} \bar{p}_{j}$ and a stochastic force $F_{j}(\bar{t})$, due to the presence of optical molasses:

$$
\begin{gathered}
\frac{d \theta_{j}}{d \bar{t}}=\bar{p}_{j}, \\
\frac{d \bar{p}_{j}}{d \bar{t}}=-\left(A e^{i \theta_{j}}+\text { c.c. }\right)-\bar{\gamma} \bar{p}_{j}+F_{j}(\bar{t}), \\
\frac{d A}{d \bar{t}}=\left\langle e^{-i \theta}\right\rangle-K A,
\end{gathered}
$$

where $j=1, \ldots, N, \bar{t}=\omega_{r} \rho t, \theta_{j}=2 k z_{j}, \bar{p}_{j}=2 k v_{z j} / \omega_{r} \rho$, and $A$ $=\left(2 \epsilon_{0} V / N \hbar \omega \rho\right)^{1 / 2} E$ are universally scaled time, position, momentum, and scattered electric field variables, respectively, normalized to the CARL bandwidth $\omega_{r} \rho$ where $\omega_{r}=2 \hbar k^{2} / m$ is the recoil frequency, $\rho$ $=\left(\Omega_{0} / 2 \Delta\right)^{2 / 3}\left(\omega d^{2} N / 2 V \hbar \epsilon_{0} \omega_{r}^{2}\right)^{1 / 3} \propto\left(P_{0} N / \Delta^{2}\right)^{1 / 3}, \quad \Omega_{0}$ is the Rabi frequency of the pump field with frequency $\omega=c k$, detuned from the atomic resonance by $\Delta=\omega-\omega_{0}, d$ is the electric dipole moment of the atom, $P_{0}$ is the intracavity pump power, and $V$ is the cavity mode volume. The average in Eq. (3) is defined as $\langle\cdots\rangle=(1 / N) \sum_{j=1}^{N}(\cdots)_{j}$. Finally, $K=\kappa_{c} / \omega_{r} \rho$ represents scaled cavity losses and $\bar{\gamma}=\gamma_{f} / \omega_{r} \rho$ is the scaled damping coefficient to account for molasses friction. With the exception of the stochastic force $F_{j}(\bar{t})$, these equations are similar to the ones used by Bonifacio and Verkerk [12] to describe CARL including the effect of collisions.

Here, the stochastic force $F_{j}(\vec{t})$ causes diffusion of the atomic momenta, i.e., heating. We assume white noise, i.e., $\left\langle F_{j}(\bar{t}) F_{j^{\prime}}\left(\bar{t}^{\prime}\right)\right\rangle=2 D_{p} \delta\left(\bar{t}-\bar{t}^{\prime}\right) \delta_{j j^{\prime}}$, where $D_{p}=\bar{\gamma} \sigma^{2}$ is the mo- 
mentum diffusion coefficient and $\sigma$ is the momentum spread in units of $\omega_{r} \rho$, corresponding to the Doppler temperature $T$ of the atoms in the molasses fields.

In the limit of strong viscous damping, it is possible to adiabatically eliminate the atomic momentum for each atom by setting $d \bar{p}_{j} / d \bar{t}=0$ in Eq. (2), so that

$$
\bar{p}_{j}=-\frac{1}{\bar{\gamma}}\left(A e^{i \theta_{j}}+\text { c.c. }\right)+\frac{F_{j}(\bar{t})}{\bar{\gamma}} .
$$

Before discussing the full model including diffusion, let us consider the simple steady-state solution neglecting the stochastic force $F$. Assuming perfect bunching, i.e., $\left\langle e^{-i \theta}\right\rangle$ $\approx e^{-i\langle\theta\rangle}$, Eqs. (3) and (4) yield [5]

$$
a=\frac{e^{-i\langle\theta\rangle}}{\kappa-i\langle p\rangle}, \quad\langle p\rangle=-\frac{2 \kappa}{\kappa^{2}+\langle p\rangle^{2}},
$$

where we have normalized all the variables in order to reduce the number of free parameters, defining $a=A / \sqrt{\bar{\gamma}}, p$ $=\sqrt{\bar{\gamma}} \bar{p}=d \theta / d \tau, \tau=\bar{t} / \sqrt{\bar{\gamma}}$, and $\kappa=\sqrt{\bar{\gamma}} K$. In the following we will use these scaled variables and parameters.

From Eqs. (5) we can identify two different steady-state regimes for CARL: when $\kappa \ll 1$ (good-cavity limit), we obtain $|\langle p\rangle| \approx(2 \kappa)^{1 / 3} \gg \kappa$ and $|a|^{2} \approx(2 \kappa)^{-2 / 3}$. From the definitions of $\kappa$ and $a$, it follows that the scattered light power $P_{s}$ is proportional to $N^{4 / 3}$ as in the usual CARL [1-3]. Conversely, for $\kappa \gg 1$ (bad-cavity limit) we obtain $|\langle p\rangle| \ll \kappa$ and $|a|^{2} \approx 1 / \kappa^{2}$, so that the scattered power $P_{s}$ is proportional to $N^{2}$, i.e., is superfluorescent.

Diffusion can be described, in the adiabatic limit of Eq. (4), writing a Fokker-Planck equation for the distribution function $P(\theta, \tau)[13,14]$. Together with Eq. (3), our model becomes

$$
\begin{gathered}
\frac{\partial P}{\partial \tau}=\frac{\partial}{\partial \theta}\left[\left(a e^{i \theta}+\text { c.c. }\right) P\right]+D \frac{\partial^{2} P}{\partial \theta^{2}}, \\
\frac{d a}{d \tau}=\int_{0}^{2 \pi} P(\theta, \tau) e^{-i \theta} d \theta-\kappa a,
\end{gathered}
$$

where $D=\sqrt{\bar{\gamma}} D_{\theta}=\sigma^{2} / \sqrt{\bar{\gamma}} \propto T\left(\Delta^{2} / \gamma_{f} N P_{0}\right)^{1 / 2}$ and $D_{\theta}=\sigma^{2} / \bar{\gamma}$ is the space diffusion coefficient. The distribution function is normalized such that $\int_{0}^{2 \pi} P(\theta, \tau) d \theta=1$ and is periodic in $\theta$ with period $2 \pi$. Hence Eqs. (6) and (7) can be written in terms of the spatial harmonics of $P(\theta, \tau)$, i.e., $P(\theta, \tau)$ $=(1 / 2 \pi) \sum_{n=-\infty}^{\infty} B_{n}(\tau) e^{i n \theta}$, where $B_{n}(\tau)=\int_{0}^{2 \pi} P(\theta, \tau) e^{-i n \theta} d \theta$, so that

$$
\begin{gathered}
\frac{d B_{n}}{d \tau}=i n\left(a B_{n-1}+a^{*} B_{n+1}\right)-n^{2} D B_{n}, \\
\frac{d a}{d \tau}=B_{1}-\kappa a .
\end{gathered}
$$

We note that $B_{-n}=B_{n}^{*}$ and $B_{0}=1$. In particular, $b=\left|B_{1}\right|$ $=\left|\left\langle e^{-i \theta}\right\rangle\right|$ is the bunching factor, describing the ampitude of the density grating. Moreover, from Eq. (4) it follows that the average momentum is $\langle p\rangle=-2 \operatorname{Re}\left(a B_{1}^{*}\right)$.

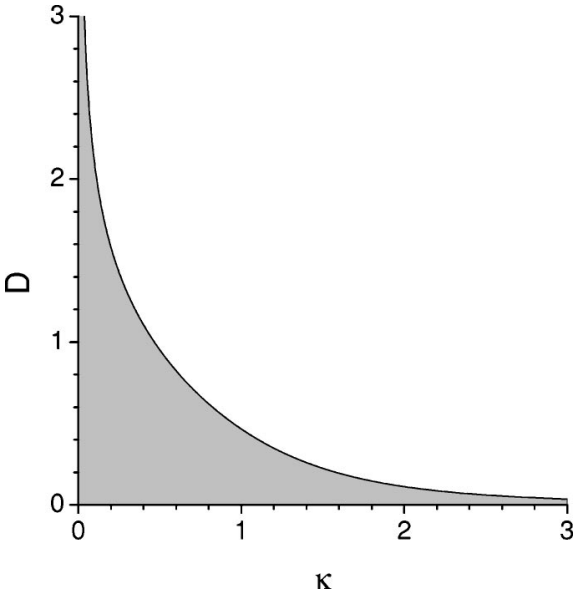

FIG. 1. Region of instability as a function of $\kappa$ and $D$ (gray area).

In the following, we will show that diffusion is responsible for the existence of a definite threshold for the steadystate solution, for which in general the bunching factor $b$ is less than 1 .

The system of Eqs. (8) and (9) have a steady-state solution with $a^{(0)}=0$ and $B_{n}^{(0)}=0$ for $n \neq 0$. If we linearize Eqs. (8) and (9) around this steady state, we find that fluctuations of $a$ and $B_{n}$ grow exponentially $\propto \exp (\lambda \tau)$ if the dispersion relation

$$
(\lambda+\kappa)(\lambda+D)=i
$$

has roots with positive real parts. The real and imaginary parts of the unstable mode of Eq. (10) gives the gain $G$ and the frequency shift $\Delta \omega$ in the exponential regime, that, in units of the cavity bandwidth $\kappa_{c}$, are

$$
\begin{gathered}
\frac{G}{\kappa_{c}}=\frac{\operatorname{Re} \lambda}{\kappa}=\frac{1}{\kappa}\left[\sqrt{\frac{C^{2}+\sqrt{1+C^{4}}}{2}}-\frac{\kappa+D}{2}\right], \\
\frac{\Delta \omega}{\kappa_{c}}=\frac{\operatorname{Im} \lambda}{\kappa}=\frac{1}{\sqrt{2} \kappa}\left[\frac{1}{\sqrt{\sqrt{1+C^{4}}+C^{2}}}\right],
\end{gathered}
$$

where $C=(\kappa-D) / 2$. The threshold condition for instability $(G \geqslant 0)$ gives rise to the following relation between $\kappa$ and $D$ :

$$
\kappa D(D+\kappa)^{2} \leqslant 1 .
$$

Figure 1 shows the regions of parameter space $(\kappa, D)$ for which Eq. (13) predicts unstable growth of the probe field and the atomic density modulation.

We note that the frequency shift at threshold (i.e., $G=0$ ) is, from Eqs. (11)-(13),

$$
\frac{\Delta \omega_{\mathrm{th}}}{\kappa_{c}}=\sqrt{\frac{D}{\kappa}}
$$

The accessible parameters in the experiment of Kruse et al. [5] are the pump power and the CARL frequency. The minimum measured value of the frequency shift was $\Delta \omega / \kappa_{c} \approx 4.6$. Assuming that it corresponds to the threshold 

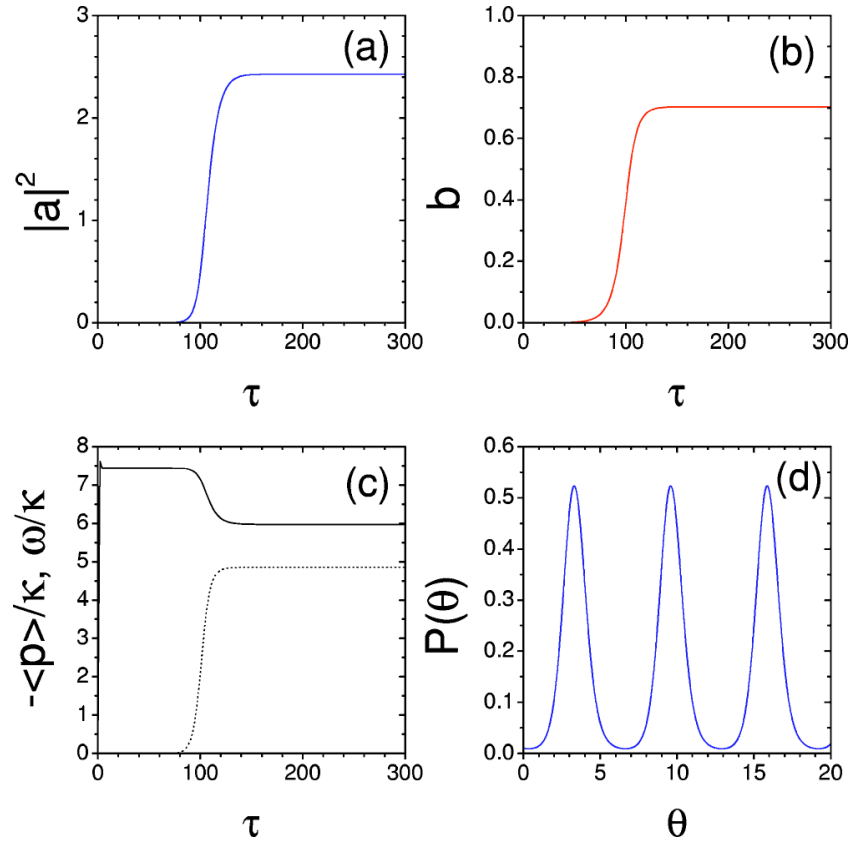

FIG. 2. Graph of $|a|^{2}$ (a), $b$ (b), $\omega / \kappa$ (continuous line) and $-\langle p\rangle / \kappa$ (dotted line) (c), vs $\tau$ and of the stationary distribution $P(\theta)$ vs $\theta(\mathrm{d})$.

value of the frequency shift, then, using Eqs. (13) and (14) we calculate that the values of $\kappa$ and $D$ at threshold are $\kappa$ $\approx 0.1$ and $D \approx 2.1$. The experiment of Ref. [5] can therefore be described using the good cavity limit where $\kappa \ll 1$. In this limit, the threshold condition Eq. (13) becomes simply $D$ $\leqslant \kappa^{-1 / 3}$, and the frequency shift at threshold is approximately $\Delta \omega / \kappa_{c} \approx \kappa^{-2 / 3} \approx D^{2}$. Using the definition of $\kappa$, the threshold value $\kappa=0.1$ can be expressed as a threshold condition for the pump power. Assuming the same parameters as in Ref. [5], i.e., $\kappa_{c}=(2 \pi) 22 \mathrm{kHz}, \gamma_{f}=9 \kappa_{c}$ and $T \approx 150 \mu \mathrm{K}$, we obtain a CARL parameter $\rho=14.6$ at threshold, corresponding to an intracavity pump power of $P_{0} \approx 3 \mathrm{~W}$, in good agreement with the experimental value.

The linear analysis can provide us with important information on the scaling behavior of the threshold input pump power and the frequency shift at threshold. Using the goodcavity limit condition $D \leqslant \kappa^{-1 / 3}$ and the scaling of $D$ and $\kappa$, the intracavity pump power scales as

$$
P_{0} \propto \frac{T^{3 / 2} \Delta^{2} \kappa_{c}^{1 / 2}}{N \gamma_{f}^{1 / 2}} .
$$

It is also worthwhile to investigate the scaling behavior of the threshold pump power and the frequency shift at threshold in the superfluorescent regime, $\kappa \gg 1$. Although to date there have been no experimental studies which operate in this regime, we can use our model to predict the behavior of such an experiment. It should be noted that from an inspection of the region of instability shown in Fig. 1, in order to operate in the superfluorescent regime and remain above the threshold for instability, it is necessary to increase scaled cavity losses $\kappa$ and decrease the scaled diffusion or temperature parameter $D$. In the bad-cavity limit the threshold con- dition Eq. (13) becomes simply $D \leqslant \kappa^{-3}$, and the frequency shift at threshold is approximately $\Delta \omega / \kappa_{c} \approx \kappa^{-2} \approx D^{2 / 3}$. Consequently the threshold pump power in the superfluorescent regime scales as

$$
P_{0} \propto \frac{T^{1 / 2} \Delta^{2} \kappa_{c}^{1 / 2} \gamma_{f}^{1 / 2}}{N} .
$$

Note that the dependence of threshold pump power on temperature is now $\propto T^{1 / 2}$ in the bad-cavity limit, as opposed to $\propto T^{3 / 2}$ in the good-cavity limit. The dependence on $N$ and $\Delta$ is the same as that for the good-cavity limit. Note that the frequency shift at threshold scales as $\sqrt{T /\left(\gamma_{f} \kappa_{c}\right)}$ in both limits.

The numerical integration of Eqs. (8) and (9) shows that the system evolves toward the stationary solution with $a^{(s)}$ $=\alpha e^{i \omega \tau}$ (where $\omega=d \phi / d \tau$ is the scaled frequency shift) and $B_{n}^{(s)}=\beta_{n} e^{i n \omega \tau}$, where $\alpha$ and $\beta_{n}$ are complex constants. As a consequence, the density distribution moves at a constant velocity, $P(\theta, \tau)=P^{(s)}(\theta+\omega \tau)$. We note that at steady state the average momentum is $\langle p\rangle=-2 \kappa b^{2} /\left(\kappa^{2}+\omega^{2}\right)$, where the bunching parameter is $b=\left|\beta_{1}\right|$. Comparing with Eq. (5), it follows that in the case of perfect bunching $(b=1) \omega=-\langle p\rangle$. Figure 2 shows the evolution of the backscattered scaled intensity $|a|^{2}(\mathrm{a})$, and the bunching $b$ (b) as a function of scaled time $\tau$ for parameters close to those of the experiment by Kruse et al., i.e., $D=1.49$, and $\kappa=0.075$. The instability was initiated using a seed field $a_{0}=10^{-5}$.

It can be seen that the field intensity and the bunching increase exponentially before relaxing to a steady state. This is the same qualitative behavior as observed in the experiment [5]. Figure 2(c) shows also the time evolution of the frequency shift $\Delta \omega / \kappa_{c}=\omega / \kappa$ (continuous line) and of the scaled mean velocity $2 k\left\langle v_{z}\right\rangle / \kappa_{c}=\langle p\rangle / \kappa$ (dashed line). We observe that the atomic mean velocity at steady state does not coincide with the velocity of the optical standing wave, i.e., $-\langle p\rangle \neq \omega$, as occurs in the case of perfect bunching $b=1$. Finally, Fig. 2(d) shows the stationary distribution $P(\theta)$ vs $\theta$, showing the density grating profile.

The steady-state solution of Eqs. (8) and (9) may be obtained solving the following recurrence equation for $\beta_{n}$ :

$$
(\omega-i n D) \beta_{n}=\alpha \beta_{n-1}+\alpha^{*} \beta_{n+1},
$$

where $n \neq 0$ and $\alpha=\beta_{1} /(\kappa+i \omega)$. Calculating $\beta_{1}$ in terms of a continued fraction and iterating numerically the solution in order to find $\omega$, the stationary solution can be obtained exactly. As an example, Fig. 3(a) shows the steady-state bunching $b$ (continuous line) and Fig. 3(b) shows $-\langle p\rangle / \kappa$ (continuous line) and $\omega / \kappa$ (dashed line) as a function of $D$ and for $\kappa=0.1$. We observe that the bunching parameter $b$ goes to zero at the threshold value $D_{t h}=2.1$, and the frequency shift at threshold is $\omega=4.6 \kappa$, in agreement with the results of the linear theory. We note from Fig. 3(b) that $-\langle p\rangle$ $\approx \omega$ only well above threshold, when $D \ll D_{t h}$.

In the experiment of Ref. [5], the pump power is ramped up and down, while the frequency shift $\omega / \kappa$ is monitored. The ramp can be designed in such a way that the pump power crosses the threshold value $P_{T}$, so that we expect that 

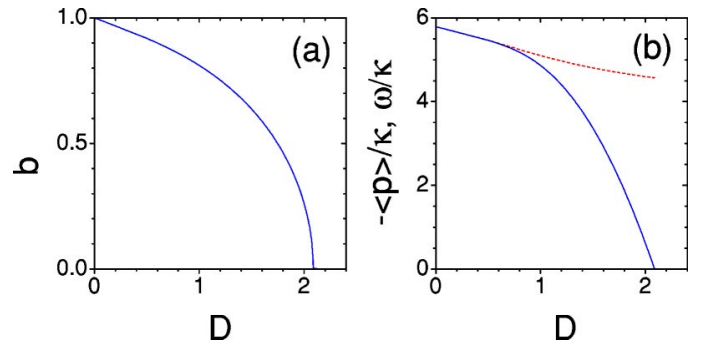

FIG. 3. (a) Steady-state solution for $b$ (continuous line). (b) Steady-state solution for $\omega / \kappa$ (dashed line) and $-\langle p\rangle / \kappa$ (continuous line), as a function of $D$ for $\kappa=0.1$.

the signal of power $P_{s}$ of the scattered light beam has the dependencies shown in Fig. 4. This behavior seems to be in agreement with preliminary experimental results, to be discussed elsewhere [10].

In conclusion, we have presented a model of the recent experiments by Kruse et al. [5] showing collective backscattering of an optical pump field by a sample of cold atoms. The model used is an adaptation of the CARL model which includes the effects of friction and diffusion forces acting on the atoms due to the presence of optical molasses fields. Using this model, we show that the system can be described by a system of coupled Maxwell-Fokker-Planck equations. It was shown using a linear stability analysis that there is a threshold condition above which collective backscattering occurs. Using a nonlinear analysis it was shown
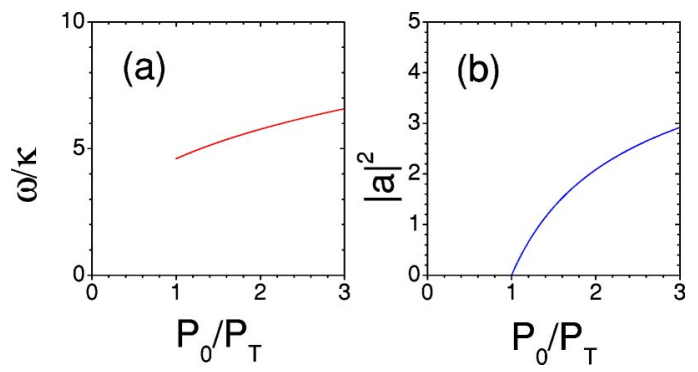

FIG. 4. Graph of frequency shift $\omega / \kappa$ (a) and scattered power $|a|^{2}$ (b) as a function of $P_{0} / P_{T}$, for $\kappa_{c}=(2 \pi) 22 \mathrm{kHz}, \gamma_{f}=9 \kappa_{c}$, and $T=150 \mu \mathrm{K}$.

that the backscattered field and atomic density modulation amplitude or "bunching" evolves to a steady state, in contrast to the nonstationary behavior observed using the usual CARL model. We suggest to test our model experimentally as well in the linear regime by searching for a threshold and characterizing its dependence from the coupling parameter and from temperature, as in the nonlinear regime. The experiment currently operates in the so-called good-cavity regime [5]. We furthermore propose to tune the experiment into the bad-cavity regime in which the atoms scatter superfluorescently, with scattered intensity $\propto N^{2}$.

The authors G.R.M.R., N.P., and R.B. would like to acknowledge support from the Royal Society of London via a European Science Exchange Joint Project.
[1] R. Bonifacio and L. De Salvo, Nucl. Instrum. Methods Phys. Res. A 341, 360 (1994); R. Bonifacio, L. De Salvo, L. M. Narducci, and E. J. D’Angelo, Phys. Rev. A 50, 1716 (1994).

[2] R. Bonifacio and L. De Salvo, Appl. Phys. B: Lasers Opt. 60, S233 (1995).

[3] R. Bonifacio, G. R.M. Robb, and B. W. J. McNeil, Phys. Rev. A 56, 912 (1997).

[4] M. Perrin, Z. Ye, and L. M. Narducci, Phys. Rev. A 66, 043809 (2002).

[5] D. Kruse, C. von Cube, C. Zimmermann, and P. W. Courteille, Phys. Rev. Lett. 91, 183601 (2003).

[6] B. Nagorny, Th. Elsässer, and A. Hemmerich, Phys. Rev. Lett. 91, 153003 (2003).

[7] H. W. Chan, A. T. Black, and V. Vuletic, Phys. Rev. Lett. 90,
063003 (2003).

[8] G. L. Lippi et al., Phys. Rev. Lett. 76, 2452 (1996).

[9] P. R. Hemmer et al., Phys. Rev. Lett. 77, 1468 (1996).

[10] C. von Cube et al. (unpublished).

[11] R. Bonifacio, P. Schwendimann, and F. Haake, Phys. Rev. A 4, 302 (1971); R. Bonifacio and L. A. Lugiato, ibid. 11, 1507 (1975).

[12] R. Bonifacio and P. Verkerk, Opt. Commun. 124, 469 (1996).

[13] F. Reif, Fundamentals of Statistical and Thermal Physics (McGraw-Hill, New York, 1985).

[14] C. Cohen-Tannoudji, Fundamental Systems in Quantum Optics, Les Houches, edited by J. Dalibard, J. M. Raimond, and J. Zinn-Justin (North-Holland, Amsterdam, 1990) p. 1. 